\title{
A PROGRAMMABLE PHASE SHIFTER FOR SINUSOIDAL SIGNALS
}

\author{
MUHAMMAD TAHER ABUELMA'ATTI and UTHMAN BAROUDI \\ King Fahd University of Petroleum and Minerals, Box 203, Dhahran 31261, Saudi \\ Arabia
}

(Received 24 January, 1996; In final form 30 August, 1996)

\begin{abstract}
A simple current(voltage)-controlled phase shifter is presented. The circuit uses a small number of active and passive components. The feasibility of obtaining a temperature-insensitive phase shifter is explored. Experimental and simulation results are included.
\end{abstract}

\section{INTRODUCTION}

Phase shifters, with accurately, stable, and reproducible phase shifts, are widely used in many areas of electronic instrumentation, telecommunication, audio-frequency measurements, and control. In an ideal phase shifter, the output would be shifted in phase from the input by a specified, adjustable angle while its magnitude would be proportional to that of the input. This can be achieved by using the traditional constant-amplitude phase shifter, shown in Fig. 1, in which the phase shift is changed manually by tuning a variable resistor[1,2]. This method, however, is not suitable for automated operation. On the other hand, digital phase shifters that transform the sinusoidal signal into a pulse signal, apply the resulting pulse signal to digital counters and finally convert the output phase-shifted pulse signal into a sinusoidal signal have been reported $[3,4]$. While these techniques are suitable for generating computer-controlled phase shifters, they are too complicated. Alternatively, an RC phase shifter, with the tunable resistor replaced by a circuit built around a digital-to-analog converter whose input resistance is digitally controlled has been proposed [5]. This method requires an excessive number of active and passive electronic 


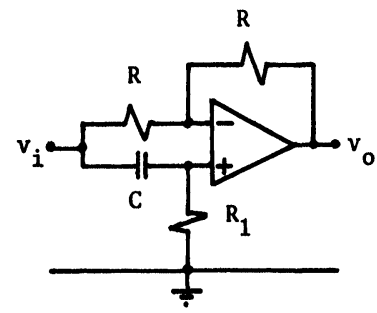

FIGURE 1 Traditional Constant-Amplitude Phase-Shifter [1]

components; namely seven operational amplifiers, a digital-to-analog converter, 14 resistors, and six capacitors. Frequency-independent phase shifters using various design techniques have also been reported [6-11]. Conventional designs using switched-capacitor circuits suffer from aliasing effects and switching transients [6-8]. Removal of these unwanted effects can be achieved, but at the expense of using additional circuitry, which results in too complicated designs. Other designs of frequency-independent phase shifters require the use of variable delay lines [9], a frequency-to-voltage converter [10], or a frequency-to-current converter [11] in addition to many resistors and capacitors, and are unattractive because of their inherent limitations [9-11].

It is the purpose of this paper to present a simple programmable phase shifter for sinusoidal signals. The proposed circuit exploits to advantage the fact that the operational transconductance amplifier (OTA) is basically a differential voltage-controlled current source in designing a voltage(current)-controlled phase shifter.

\section{PROPOSED CIRCUIT}

Figure 2 shows the proposed voltage(current)-controlled phase shifter. Assuming an ideal operational amplifier with infinite input impedance, zero output impedance and infinite differential gain, and an ideal OTA with $I_{0}=g_{m}\left(V_{+}-V_{-}\right)$, infinite input impedance and zero output impedance, routine analysis yields the transfer function of the circuit of Fig. 2, which can be expressed as

$$
T(s)=\frac{v_{o}}{v_{i}}=-\frac{1+g_{m} R_{B}-s C\left(R_{A}+R_{B}\right)}{1+g_{m} R_{B}+s C\left(R_{A}+R_{B}\right)}
$$




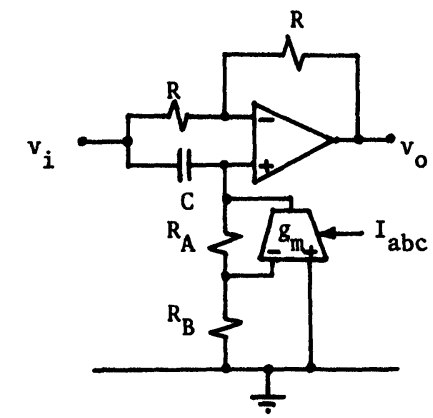

FIGURE 2 Proposed Voltage (Current)- Controlled Phase Shifter

Equation (1) corresponds to the transfer function of a first-order all-pass filter, or phase shifter, with a unity-gain and a phase shift, between the input voltage and the output voltage of

$$
\Phi=\pi-2 \tan ^{-1} \frac{\omega C\left(R_{A}+R_{B}\right)}{1+g_{m} R_{B}}
$$

Equation (2) shows that the phase shift can be easily controlled by adjusting the transconductance $g_{m}$ of the OTA. For a bipolar OTA, the transconductance $g_{m}$ can be expressed as

$$
g_{m}=\frac{I_{a b c}}{2 V_{T}}
$$

where $I_{a b c}$ is the auxiliary bias current of the OTA and $V_{T}$ is the thermal voltage $\cong 26 \mathrm{mV}$ at room temperature. From (2) and (3), it can be seen that the phase shift can be adjusted by controlling the current $I_{a b c}$. This current can be obtained from the output of a digital-to-analog converter (DAC). Thus the realization of a digitally programmable sinusoidal phase shifter is feasible. But such a realization suffers from the temperature dependence of the phase shift. This is attributed to the dependence of $V_{T}$, and consequently $g_{m}$, on the temperature. However, a temperature insensitive phase shifter can be realized if the resistance $R_{B}$ is replaced by an OTA-based grounded-resistance realization as shown in Fig. 3. Re-analysis yields the transfer function of the circuit of Fig. 3, which can be expressed as

$$
\Phi=\pi-2 \tan ^{-1} \frac{\omega C\left(g_{m 2} R_{A}+1\right)}{g_{m 1}+g_{m 2}}
$$

where $g_{m i}, i=1,2$ is the transconductance of the ith OTA. If we choose $g_{m 2} R_{A}$ » 1 , then (4) reduces to

$$
\Phi=\pi-2 \tan ^{-1} \frac{\omega C R_{A}}{1+g_{m 1} / g_{m 2}}=\pi-2 \tan ^{-1} \frac{\omega C R_{A}}{1+I_{a b c 1} / I_{a b c 2}}
$$


From (5), it can be seen that the phase shift is temperature-insensitive and can be controlled by adjusting the current $I_{a b c 1}$ while the current $I_{a b c 2}$ is kept constant and satisfying the condition $I_{a b c 2} \gg \frac{2 V_{T}}{R_{A}}$

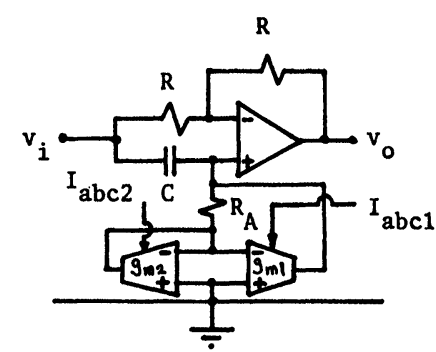

FIGURE 3 Proposed Temperature-Insensitive Current (Voltage)-Controlled Phase Shifter

\section{EXPERIMENTAL AND SIMULATION RESULTS}

The proposed phase shifter circuit of Fig. 2 was experimentally tested using the uA741 operational amplifier (OA) and the LM 13600 OTA. The results obtained with $R=5 \mathrm{~K}, C=10 \mathrm{nF}, R_{A}=7.62 \mathrm{~K}$, and $R_{B}=1 \mathrm{~K}$ are shown in Fig. 4. From Fig. 4, it can be seen that the measured results are in good agreement with the presented theory. The temperature-insensitive phase shifter of Fig. 3 was simulated using the SPICE circuit simulation program. The OTAs were modelled using the model of Fig. 5 [12] and the OA was modelled using the uA741 model available in the file eval.lib of SPICE. The results obtained with $R_{A}=10 \mathrm{~K}, C=10 \mathrm{nF}, R=5 \mathrm{~K}$, and $I_{a b c 2}=$ $250 \mu A$ are summarized in Fig. 6. From Fig. 6, it can be seen that the simulation results are in good agreement with the theory presented.

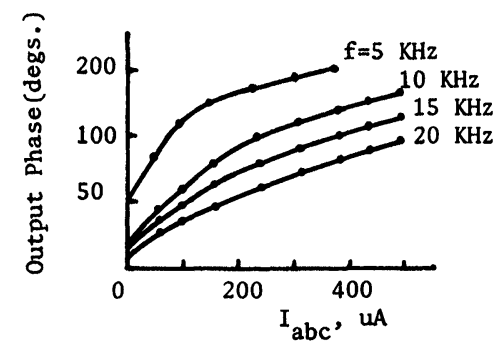

FIGURE 4 Experimental Results obtained from the Circuit of Fig. 2 with: $\mu$ A741 0A; $\underline{\mathrm{LM} 13600}$ OTA, $\mathrm{R}=5 \mathrm{~K} ; \mathrm{C}=10 \mathrm{nF} ; \mathrm{R}_{\mathrm{A}}=7.62 \mathrm{~K}$. 


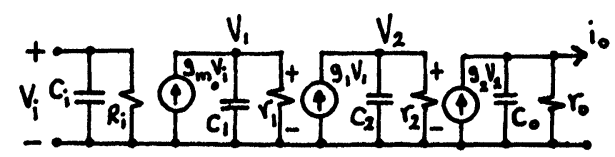

FIGURE 5 The OTA model used in Simulation [12] $\mathrm{gm}_{\mathrm{o}}=1923 \mu \mathrm{s} ; \mathrm{g}_{1}=1 / \mathrm{r}_{1}=1000 \mu \mathrm{S} ; \mathrm{g}_{2}=$ $1 / \mathrm{r}_{2}=1000 \mu \mathrm{S} ; \mathrm{C}_{\mathrm{i}}=4.5 \mathrm{PF} ; \mathrm{C}_{1}=25.3 \mathrm{PF} ; \mathrm{C}_{2}=2.53 \mathrm{PF} ; \mathrm{C}_{\mathrm{o}}=7.5 \mathrm{PF} ; \mathrm{R}_{\mathrm{i}}=65 \mathrm{~K} ; \mathrm{R}_{\mathrm{o}}=63 \mathrm{M} \Omega$

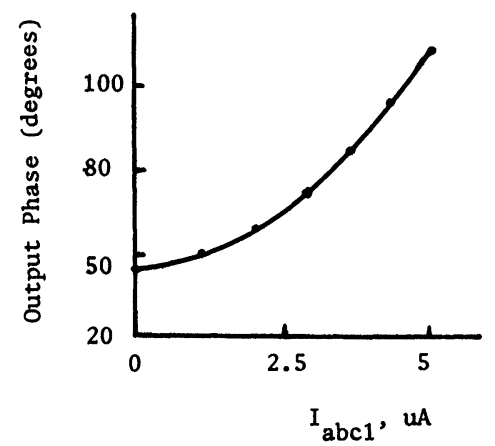

FIGURE 6 Simulation Results obtained from the Circuit of Fig. 4 with: $R A=10 \mathrm{~K} ; R=5 \mathrm{~K}$; $\mathrm{C}=10 \mathrm{nF} ; \mathrm{f}=1 \mathrm{KHz}, \mathrm{Iabc}_{2}=250 \mu \mathrm{A}$.

\section{CONCLUSION}

In this paper, a simple current(voltage)-controlled temperature-insensitive phase shifter has been presented. The circuit uses only two OTAs, one OA, three resistors, and one capacitor. The circuit can provide a continuous control on the phase shift rather than the coarse change obtainable from most of the digitally programmable phase shifters.

\section{Acknowledgements}

The authors acknowledge valuable discussions with Dr. A.R. Al-Ali.

\section{References}

[1] A.S. Sedra and K.C. Smith, Microelectronic Circuits, Saunders College Publishing, Philadelphia:1991

[2] S.V. Cherepov, Bridge for ac measurement of magnetic susceptibility, Instruments and Experimental Techniques, Vol.25, \#5, Part2, 1986, pp.1226-1227

[3] V.S. Batrachenko and T.V. Zarubina, Controllable digital phase shifter, Instruments and Experimental Techniques, Vol.21, \#5, 1978, pp.1344-1345 
[4] N.V. Maslov and R.P. Prikhnenko, Digital phase shifter for a precision calibrator of phase shifts, Instruments and Experimental Techniques, Vol.21, \#5, 1978, pp.13461347

[5] D.A. Lapshin, A digitally controlled phase shifter for sinusoidal signals, Instruments and Experimental Techniques, Vol.37, \#1, Part2, 1994, pp.76-77

[6] M. Mittal and S.S. Jamuar, Programmable frequency-independent switched-capacitor phase shifter of unity gain, Measurement Science and Technology, Vol.2, 1991, pp. $475-477$

[7] A. Helfrick, Phase shifter uses digital techniques, EDN, Vol.32, November 12,1987, pp. 289-290

[8] B.K. Jones, B.K. Sharma and D.H. Bidle, A frequency-independent phase shifter, Journal of Physics E: Scientific Instruments, Vol.13, 1980, pp.1346-1347

[9] B. Cowan, A versatile phase shifter for NMR experiments and other applications, Measurement Science and Technology, Vol.3, 1992, pp.296-298

[10] M. Madihian, K. Watanabe and T. Yamamoto, A frequency-independent phase shifter, Journal of Physics E: Scientific Instruments, Vol.12, 1979, pp.1031-1032

[11] L. Bruschi, R. Storti and G. Torzo, Simple design for a frequency-independent voltagecontrolled phase shifter, Review Scintific Instruments, Vol.58, 1987, pp.1960-1962

[12] P. Bowron and H.M. Dahir, Modelling of non-ideal active devices in continuous time OTA-C filters, European Conference on Circuit Theory and Design, ECCTD 89, IEE Conference Publication \#308, 1989, pp.128-131 

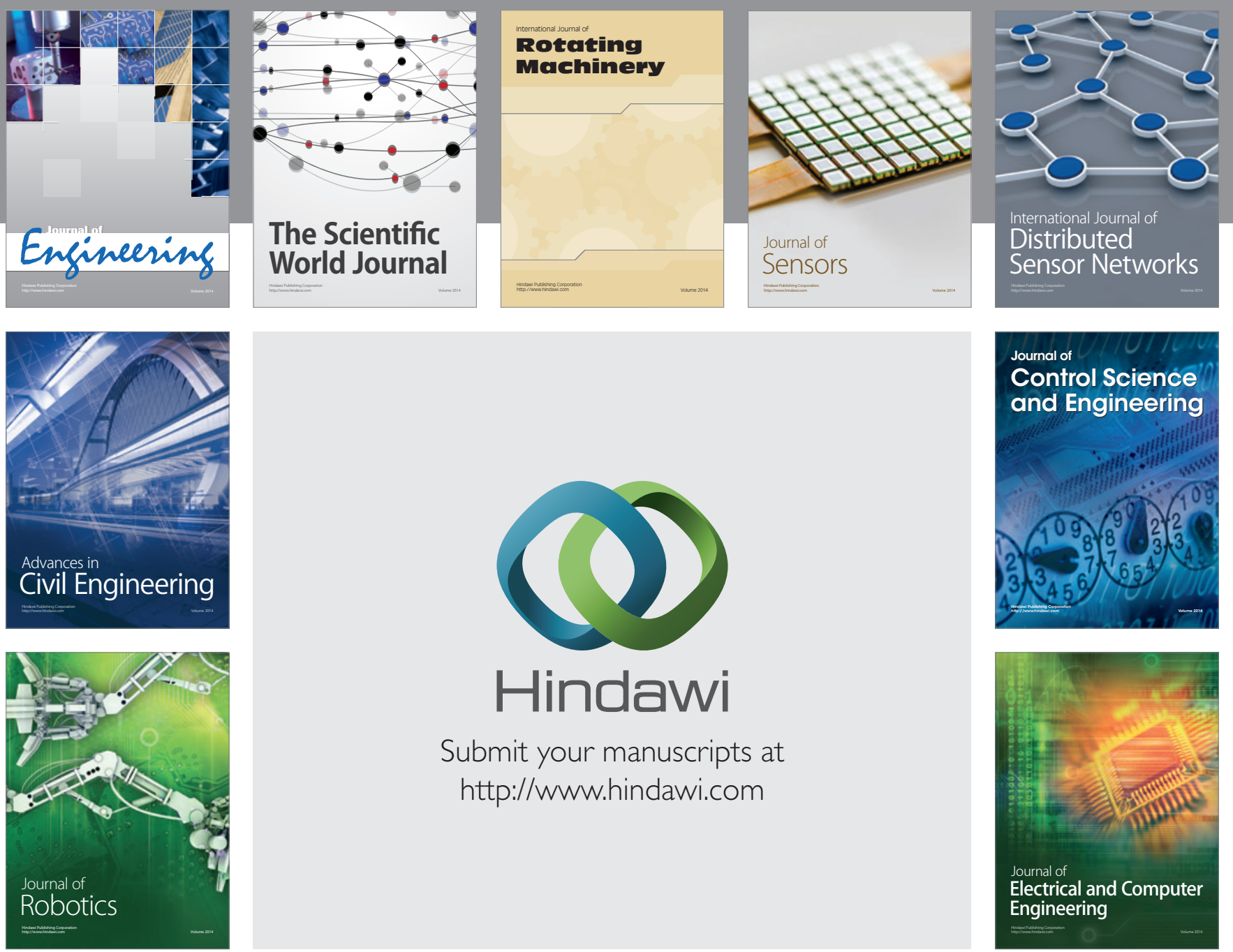

Submit your manuscripts at

http://www.hindawi.com
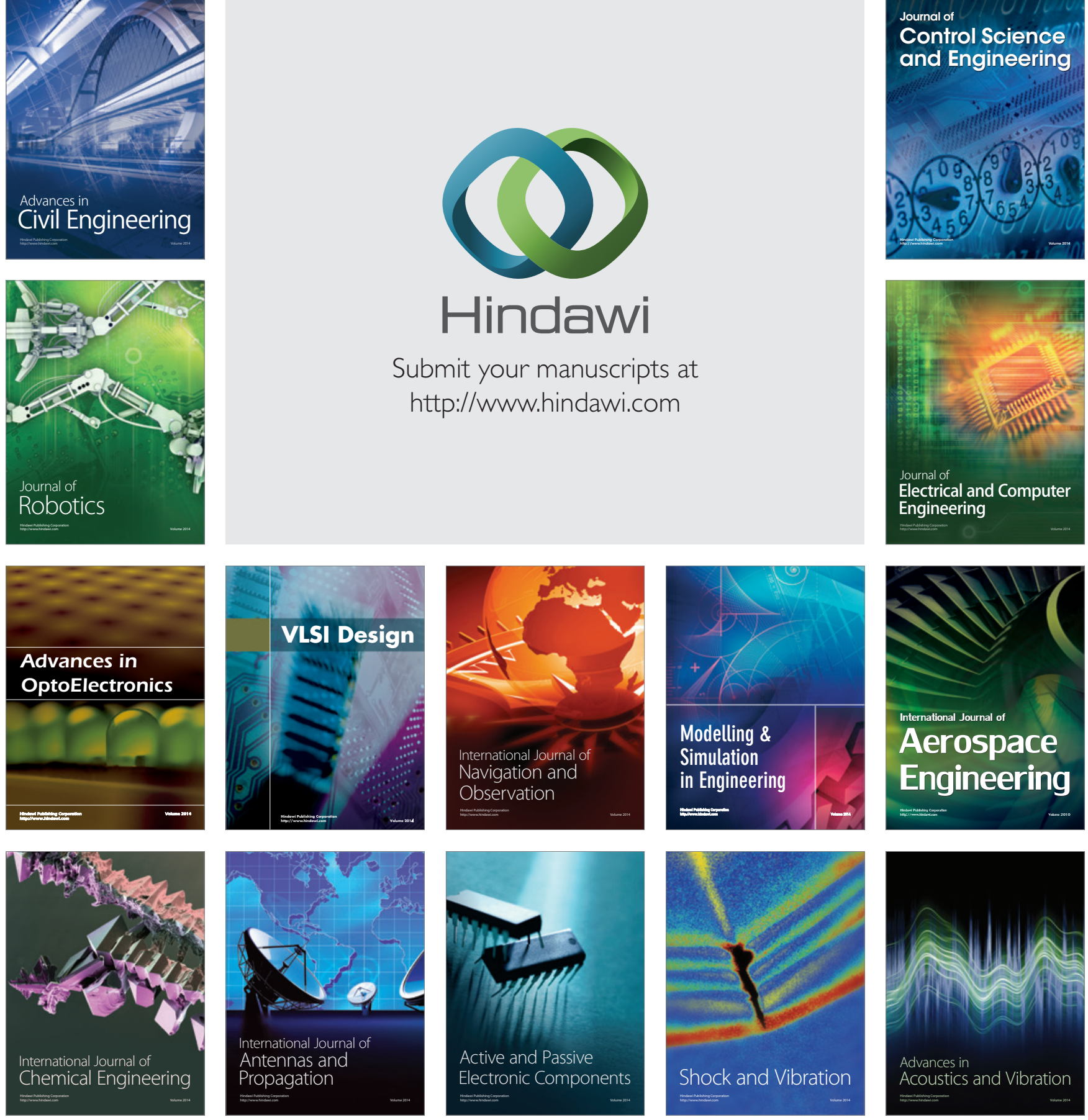